\title{
Tuberculose péritonéale
}

\author{
Imhokhai Ogah MD, Fiona Milne MD, Boris Zevin MD PhD
}

Citation : CMAJ 2021 November 1;193:E1664. doi : 10.1503/cmaj.210355-f

Voir la version anglaise de l'article ici : www.cmaj.ca/lookup/doi/10.1503/cmaj.210355

$\mathbf{U}$

ne femme de 66 ans, auparavant en bonne santé, a consulté à l'hôpital pour malaise, ballonnements et satiété rapide depuis un mois. Originaire des Philippines, elle a immigré il y a 40 ans et a déjà été exposée à la tuberculose (TB); un test de libération d'interféron gamma s'est révélé positif. La tomodensitométrie abdominale a montré la présence d'ascite et de nodules épiploïques.

La paracentèse diagnostique a confirmé une ascite principalement lymphocytaire avec un gradient sérumascite d'albumine bas, sans cellules cancéreuses. Les tests de coloration étaient négatifs pour les bacilles acido-résistants et les résultats des cultures étaient négatifs pour les mycobactéries. Nous avons effectué une laparoscopie diagnostique qui a montré un péritoine pariétal et viscéral constellé de dépôts blancs, concordant avec une TB péritonéale (figure 1A). Les spécimens de biopsie péritonéale ont confirmé des granulomes non caséifiés; mis en culture, ils ont révélé une pansensibilité pour le complexe Mycobacterium tuberculosis (figure 1B) en restant néanmoins négatifs pour ce qui est de ces organismes. Après une antibiothérapie appropriée, l'état clinique de la patiente s'est amélioré. Nous avons signalé le cas aux autorités de santé publique pour une recherche de contacts.

L'incidence de la TB active au Canada est de 4,9/1000001. Les facteurs de risque à l'égard de l'infection incluent: immunosuppression, contact avec des cas soupçonnés ou avérés, séjour dans des pays où la maladie est endémique, immigration à partir de ces pays ou appartenance à des communautés où la prévalence de TB est élevée ${ }^{1}$. Quatre-vingts pour cent des personnes qui souffrent de TB ont une atteinte pulmonaire; la TB péritonéale isolée ne s'observe que dans $1 \%$ des $\operatorname{cas}^{2,3}$. Le diagnostic est complexe, car la coloration des bacilles acido-résistants et la culture mycobactérienne de l'ascite peuvent avoir des sensibilités d'aussi peu que $3 \%$ et $20 \%$ ou moins, respectivement ${ }^{4-6}$. La culture des spécimens de biopsie péritonéale pour recherche de mycobactéries est l'épreuve diagnostique standard. Utilisé avec l'histologie, l'examen laparoscopique présente des sensibilités et spécificités supérieures à $90 \% \%^{5}$. La tuberculose péritonéale devrait faire partie du diagnostic différentiel de l'ascite en présence d'un gradient sérum-ascite d'albumine bas avec lymphocytose chez des patients exposés à des facteurs de risque ou présentant une infection latente connue.

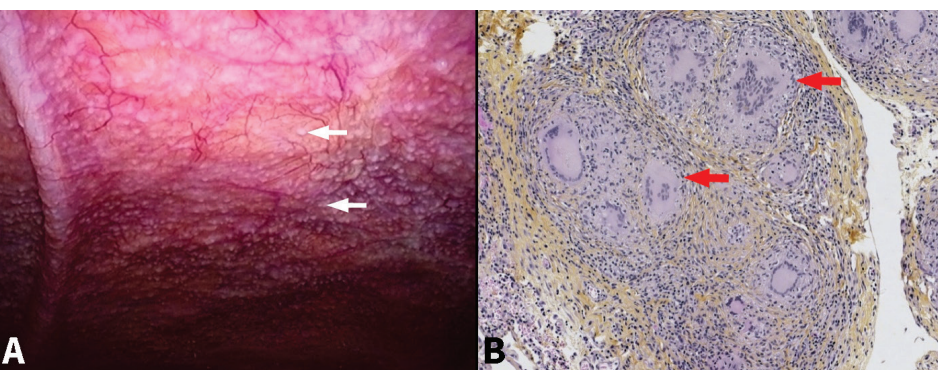

igure 1 : Cliché laparoscopique chez une femme de 66 ans souffrant de tubercuose péritonéale. A) Photographie montrant des dépôts tuberculeux blancs (flèches blanches) au péritoine pariétal. B) Cliché au microscope d'un échantillon de biopsie péritonéale montrant de nombreux granulomes non nécrosants (flèches rouges). Coloration à l'hématoxyline/phloxine/safran. Amplification originale × 100 .

\section{Références}

1. Tuberculosis: Monitoring. Ottawa: Public Health Agency of Canada; modified 2019 Mar. 25. Accessible ici : https://www.canada.ca/en/public-health/ services/diseases/tuberculosis.html (consulté le 28 avr. 2021).

2. Natali D, Cloatre G, Brosset C, et al. What pulmonologists need to know about extrapulmonary tuberculosis. Breathe (Sheff) 2020;16:200216.

3. Reported tuberculosis in the United States, 2017. Atlanta: Centers for Disease Control and Prevention; reviewed 2018 Oct. 22. Accessible ici : https://www. cdc.gov/tb/statistics/reports/2017/default.htm (consulté le 12 févr. 2021).

4. Sanai FM, Bzeiki KI. Systematic review: tuberculous peritonitis - presenting features, diagnostic strategies and treatment. Aliment Pharmacol Ther 2005;22: 685-700.

5. Debi U, Ravisankar V, Prasad KK, et al. Abdominal tuberculosis of the gastrointestinal tract: revisited. World J Gastroenterol 2014;20:14831-40.

6. Koff A, Azar MM. Diagnosing peritoneal tuberculosis. BMJ Case Rep 2020; 13:e233131.

Intérêts concurrents : Aucun déclaré.

Cet article a été révisé par des pairs.

Les auteurs ont obtenu le consentement de la patiente.

Affiliations : Départements de médecine interne (Ogah, Milne) et de chirurgie (Zevin), Université Queen's, Kingston, Ont.

Propriété intellectuelle du contenu : Il s'agit d'un article en libre accès distribué conformément aux modalités de la licence Creative Commons Attribution (CC BY-NC-ND 4.0), qui permet l'utilisation, la diffusion et la reproduction dans tout médium à la condition que la publication originale soit adéquatement citée, que l'utilisation se fasse à des fins non commerciales (c.-à-d., recherche ou éducation) et qu'aucune modification ni adaptation n'y soit apportée. Voir : https://creativecommons.org/licenses/by-nc-nd/4.0/deed.fr.

Correspondance : Imhokhai Ogah, i.ogah@queensu.ca 